\title{
碱在过渡金属催化的有机化学反应中的作用
}

\author{
欧阳昆冰 ${ }^{a}$ 席振峰*, \\ $\left({ }^{a}\right.$ 中国科学院化学研究所 北京 100190) \\ $\left({ }^{b}\right.$ 北京大学化学学院 北京 100871)
}

\begin{abstract}
摘要 碱, 包括无机碱和有机碱, 在过渡金属催化的有机反应中发挥着重要作用. 本文概括地介绍和讨论相关文献中 碱的作用机制. 碱的作用与许多因素有关，包括：碱性、溶解度、电离度、溶剂、聚集度、金属离子大小、金属离子 Lewis 酸性、金属离子的 “软硬” 度、阴离子的大小、阴离子的配位作用等. 碱可用于㩲取质子、中和反应体系中的 酸、活化催化剂、促进催化剂再生等, 碱中金属阳离子的作用主要集中在其对碱在有机溶剂中溶解度的影响和其与底 物或溶剂间相互作用力的强弱上，碱中阴离子的作用主要表现在离子与金属的配位方式和稳定性上; 有机碱和无机碱 的主要区别体现在溶解度和空间位阻的不同上. 另外, 商品碱中极少量的过渡金属杂质也有可能对反应产生影响.
\end{abstract}

关键词 碱的作用; 碱性; 无机碱; 有机碱; 过渡金属催化的有机反应

\section{Roles of Bases in Transition-Metal Catalyzed Organic Reactions}

\author{
Ouyang, Kunbing $^{a} \quad \mathrm{Xi}$, Zhenfeng ${ }^{*, b}$ \\ ( ${ }^{a}$ Institute of Chemistry, Chinese Academy of Sciences (CAS), Beijing 100190) \\ ( ${ }^{b}$ College of Chemistry, Peking University, Beijing 100871)
}

\begin{abstract}
Bases, either organic bases or inorganic ones, are very often added in transition-metal catalyzed organic reactions to promote the catalytic reaction efficiency and increase the yields of products. As a common practice for most published papers, a reaction condition screening table is given, listing a number of bases and the respective yield of products. However, no discussion or a little in some cases is provided on why one base works well to give a high yield formation of the product, but other bases afford no or low yields of the product. Furthermore, in many cases a certain base works well for one reaction but may not work for another. Indeed, for a complicated reaction mixture containing several different components, it is very difficult to analyze and understand the roles of certain bases. Yet there are sporadic discussions and rationalization on the roles of bases in the literature. The role of bases has been reported to be straightforward in some cases, for examples, to abstract protons or to neutralize acids in the reaction system, but very complicated in many other cases. The roles of bases may be affected by several factors, including basicity, solubility, ionization ability, solvent, aggregation state, the size of the metal cations, Lewis acidity of the metal cations, the HSAB theory, the size of the counter anions; the coordination ability of the counter anions, etc. In addition to abstract protons or to neutralize acids in the reaction system, a base may activate the catalysts and facilitate the regeneration of reactive catalytic species. The roles of the metal cation in a base may mainly influence the solubility of the base in organic solvents, or interact with substrates or solvents. The roles of the counter anion in a base may mainly contribute to the coordination with a metal center and subsequent stabilize the complex. The major differences between inorganic bases and organic bases include their solubility and bulkiness. Metal contaminants in bases may also have innegligible effect on the reactions, since in many cases a large excess amount of bases are added. This review is written based on the limited knowledge, focusing on commonly used inorganic bases such as LiOBu- $t$, NaOBu- $t$, KOBu- $t$, LiOAc, $\mathrm{NaOAc}, \mathrm{KOAc}, \mathrm{LiOH}, \mathrm{NaOH}, \mathrm{KOH}, \mathrm{Li}_{2} \mathrm{CO}_{3}, \mathrm{Na}_{2} \mathrm{CO}_{3}, \mathrm{~K}_{2} \mathrm{CO}_{3}, \mathrm{Cs}_{2} \mathrm{CO}_{3}, \mathrm{KF}$, CsF and organic bases such as DBU and $\mathrm{Et}_{3} \mathrm{~N}$. Keywords roles of bases; basicity; inorganic bases; organic bases; transition-metal catalyzed organic reactions
\end{abstract}

\section{1 引言}

许多过渡金属催化的有机反应由于碱的加入，反应 效率大大提高, 产物收率大大提高. 因此, 在相关文献 中, 经常看到反应条件优化的表格, 有的碱如 LiOBu- $t$ 可以使产物收率很高, 但是其他碱如 KOBu- $t$ 则效果差、 产物收率低, 或者完全没有效果. 许多文献以及我们自
己的研究结果显示(图 1) ${ }^{[1]}$, 一个特定的碱在一个反应中 效果很好, 但是在另外一个反应中则完全没有效果, 或 者导致不同类型产物的生成. 虽然很多研究表明碱在过 渡金属催化的有机反应中很重要, 文献中也有零星的讨 论，但是还没有看到文献概括地讨论过渡金属催化有机 反应中碱的作用机制, 以及为什么不同的碱甚至看上去

\footnotetext{
* E-mail: zfxi@pku.edu.cn; Tel.: 010-62759728

Received November 29, 2012; published December 20, 2012.

Project supported by the National Natural Science Foundation of China (No. 21132001) and the 973 program (No. 2012CB821603).

项目受国家自然科学基金(No. 21132001)和 973 项目(No. 2012CB821603)资助.
} 
类似的碱会产生很不同的效果. 事实上, 通过我们自己 的研究以及阅读有关文献, 我们意识到碱的作用机制很 复杂, 与多种因素有关, 包括: 碱性、溶解度、电离度、 聚集度、溶剂、金属离子大小、金属离子 Lewis 酸性、 金属离子的 “软硬” 度、阴离子的大小、阴离子的配位 作用等. 碱可用于篗取质子、中和反应体系中的酸、活 化催化剂、促进催化剂再生等; 碱中金属阳离子的作用 主要集中在其对碱在有机溶剂中溶解度的影响和其与 底物或溶剂间相互作用力的强弱上; 碱中阴离子的作用 主要表现在离子与金属的配位方式和稳定性上; 有机碱 和无机碱的主要区别体现在溶解度和空间位阻的不同 上. 另外, 由于反应中碱的加入量往往过量甚至过量数 倍，因此商品碱中极少量的过渡金属杂质也将可能对反 应产生影响, 从而使碱的作用机制产生假象, 给研究带 来更多困难. 本文试图将文献中有关过渡金属催化有机 反应中碱的作用机制的讨论进行归纳和概括, 重点关注 了常见的 LiOBu- $t$, NaOBu- $t$, KOBu- $t$, LiOAc, NaOAc, $\mathrm{KOAc}, \mathrm{LiOH}, \mathrm{NaOH}, \mathrm{KOH}, \mathrm{Li}_{2} \mathrm{CO}_{3}, \mathrm{Na}_{2} \mathrm{CO}_{3}, \mathrm{~K}_{2} \mathrm{CO}_{3}$, $\mathrm{Cs}_{2} \mathrm{CO}_{3}, \mathrm{KF}, \mathrm{CsF}$ 等无机碱以及 $\mathrm{DBU}$ 和 $\mathrm{NEt}_{3}$ 等有机碱.

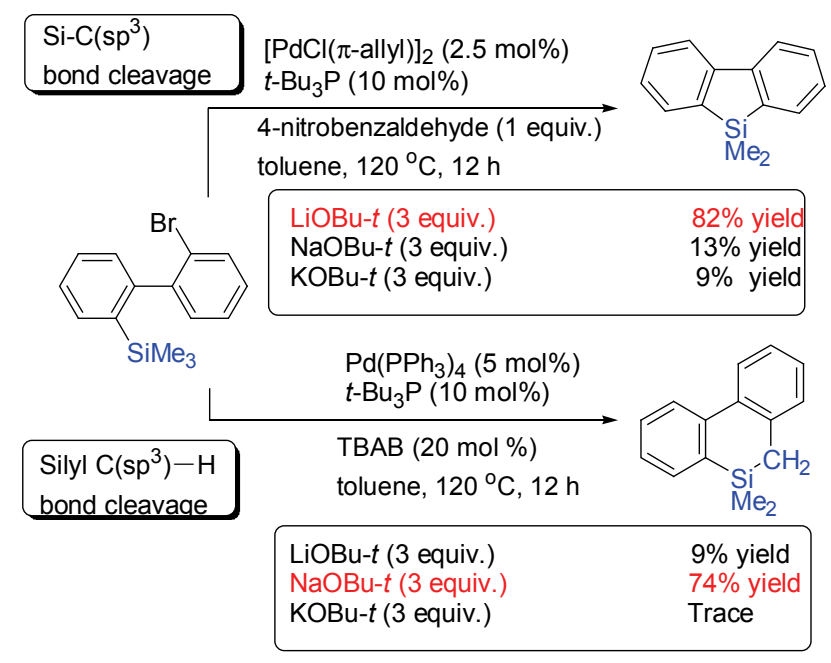

图 1 三甲基硅基中碳一硅键或碳一氢键的选择性催化切断中碱的作 用 ${ }^{[1]}$

Figure 1 Roles of bases in transition-metal catalyzed selective cleavage of $\mathrm{Si}-\mathrm{C}$ bond or $\mathrm{C}-\mathrm{H}$ bond in $\mathrm{SiMe}_{3}$ group $^{[1]}$

\section{2 碱性、溶解度和溶剂的影响}

目前, 大部分的研究认为碱在化学反应中的主要作 用是去质子化和中和体系中的质子或酸. 碱的去质子化 能力与其 $\mathrm{p} K_{\mathrm{a}}$ 值 (无机碱的共轭酸的 $\mathrm{p} K_{\mathrm{a}}$ 值)及其在相应 溶剂中的溶解度有着十分紧密的联系.

对不同碱在不同有机溶剂中的溶解度进行专门研 究的文献极少 $(\text { 表 } 1)^{[2]}$. 无机碱在有机溶剂中的碱性并不 好衡量(图 2) ${ }^{[3]}$, 这是因为大部分的无机碱在有机溶剂中 的溶解性都不是很好, 同时, 所使用的溶剂和金属离子
在溶剂中的聚集度也会对碱的性质带来影响. 另一方 面, 由于溶剂具有拉平效应，不同溶剂的分辨宽度也不 同, 因此研究无机碱在有机溶剂中的碱性是一项比较复 杂的工作. 根据已有的文献报道, 在多数有机溶剂中, $\mathrm{Cs}_{2} \mathrm{CO}_{3}$ 与 $\mathrm{DBU}$ 的碱性差不多, 而 $\mathrm{KOH}$ 与 $\mathrm{NaOBu}-t$ 的 碱性相当 ${ }^{[3,4]}$.

表 1 碳酸盐在一些偶极非质子溶剂中的溶解度 ${ }^{a}$

Table 1 Solubility of metal carbonate salts in dipolar aprotic solvents

\begin{tabular}{ccccc}
\hline Solvent & $\mathrm{Li}_{2} \mathrm{CO}_{3}$ & $\mathrm{Na}_{2} \mathrm{CO}_{3}$ & $\mathrm{~K}_{2} \mathrm{CO}_{3}$ & $\mathrm{Cs}_{2} \mathrm{CO}_{3}$ \\
\hline DMF & 0.003 & 0.038 & 0.075 & 1.195 \\
$\mathrm{Me}_{2} \mathrm{SO}$ & 0.014 & 0.143 & 0.470 & 3.625 \\
DMAC & 0.004 & 0.021 & 0.046 & 0.490 \\
Sulfolane & 0.021 & 0.031 & 0.160 & 3.950 \\
NMP & 0.014 & 0.208 & 0.237 & 7.224 \\
\hline
\end{tabular}

${ }^{a}$ Solubilities in $\mathrm{g} / 10 \mathrm{~mL}$ determined at ambient temperature by flame photometry.

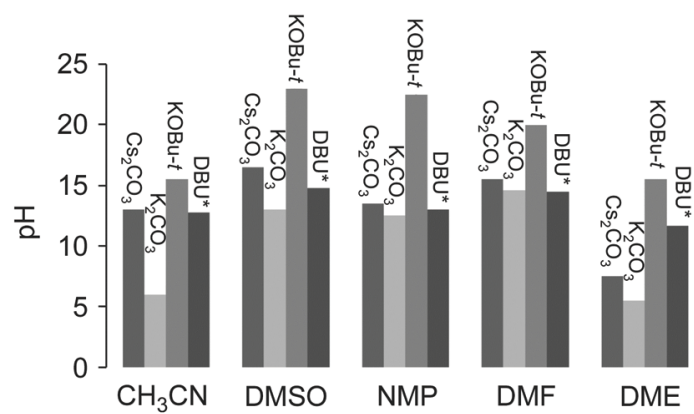

图 2 碳酸铯、碳酸钾、叔丁醇钾和 DBU 在一些非质子溶剂中的碱 性 ${ }^{[3]}$

Figure 2 Basicities of cesium and potassium carbonates, potassium tert-butoxide and DBU in various aprotic solvents ${ }^{[3]}$

$5 \mathrm{mmol} / 100 \mathrm{~mL}$ weight-in quantity, $*=$ complete dissolution

反应中使用的碱的溶解度和反应体系的碱性强弱 都可能对反应结果产生明显影响. Kobayashi 等 ${ }^{[5]}$ 在 2012 年报道了叔丁醇锂作用下的吲哚 3 位羧基化反应(图 3). 该反应最初使用的是钯催化的体系, 反应中选择不同碱 会使得反应收率有明显变化. 当使用弱碱 $\mathrm{K}_{2} \mathrm{CO}_{3}$ 时能得 到痕量的羧基化产物, 而当使用较强的 $\mathrm{Cs}_{2} \mathrm{CO}_{3}$ 时, 反应 收率有了显著提高. 碱性强弱的影响同样体现在叔丁醇 类碱中一一使用碱性较弱的叔丁醇锂能得到好的反应 收率但碱性相对较强的叔丁醇钠则不能得到预期产物. 尽管进一步的研究表明, 该反应不需要金属催化剂也能 顺利进行，但相关结果仍表明碱对反应收率有影响. 作 者也对此提出了可能的反应机理一一叔丁醇锂首先与 底物 1 中酸性最强的 $\mathrm{N}-\mathrm{H}$ 键反应, 得到锂化物 2. 若这 一物种直接被 $\mathrm{CO}_{2}$ 捕捉, 则得到 5 . 尽管 $\mathrm{N}$ 上的亲核取 代是更加有利的，但当反应温度较高时， $2,3,4$ 之间的 可逆过程会促使产物 6 的生成. 碱性太强的碱可能比较 倾向于生成 N-1 位反应的产物. 

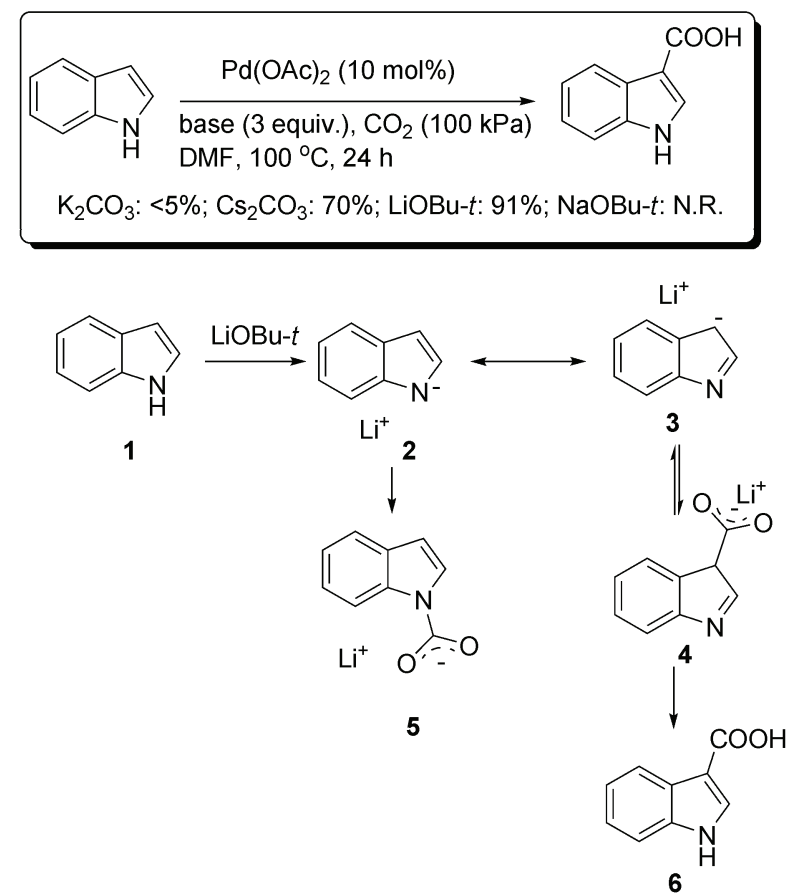

图 3 叔丁醇锂作用下的品哚 3 位羧基化反应 ${ }^{[3]}$

Figure 3 Direct carboxylation of indole mediated by LiOBu- $t^{[5]}$

碱在溶液中的状态也会影响反应的结果. 刘否等 ${ }^{[6]}$ 为解决反应中无机碱在有机溶剂中的溶解度不好的问 题, 设计合成了离子型有机碱(由大位阻有机阳离子和 碱性阴离子组成, 如图 4 所示). 作者通过测定这些离子 型有机碱在部分代表性有机溶剂中的电导率, 并与一些 常见无机碱的相应电导率进行比较发现(表 2$)^{[6]}$, 在特定 反应体系中溶解度好的碱并不一定是有效的碱, 较强的 离子化能力是离子型有机碱表现优异的主要原因.

在众多的偶联反应中, 碱都是一个不可或缺的组 分. 大部分钯催化的偶联反应中, 活性催化物种都是 $\operatorname{Pd}(0)$, 但在多数反应中, 实际加入的催化剂是 $\operatorname{Pd}(\mathrm{II})$. 在 $\operatorname{Pd}(\mathrm{II})$ 向 $\operatorname{Pd}(0)$ 转化的过程中, 需要碱的加入. 尽管这 个过程的具体机理目前尚未明确, 但也有一些关于活性 催化物种生成机理方面的研究.

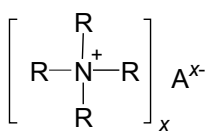

TMAP: $\mathrm{R}=\mathrm{Me}, \mathrm{A}^{\mathrm{x}-}=\mathrm{PO}_{4}^{3-}$

TBAP: $\mathrm{R}=n-\mathrm{Bu}, \mathrm{A}^{\mathrm{x}-}=\mathrm{PO}_{4}^{3-}$

TBAC: $\mathrm{R}=n-\mathrm{Bu}, \mathrm{A}^{\mathrm{x}-}=\mathrm{CO}_{3}^{2-}$

TBAE: $\mathrm{R}=n-\mathrm{Bu}, \mathrm{A}^{\mathrm{x}-}=\mathrm{OAC}^{-}$

TBAA: $\mathrm{R}=n-\mathrm{Bu}, \mathrm{A}^{x-}=$ adipate

TEAP: $\mathrm{R}=\mathrm{Et}, \mathrm{A}^{\mathrm{x}-}=\mathrm{PO}_{4}^{3-}$

TBAHC: $\mathrm{R}=n-\mathrm{Bu}, \mathrm{A}^{x-}=\mathrm{HCO}_{3}^{-}$

TBAO: $\mathrm{R}=n-\mathrm{Bu}, \mathrm{A}^{x-}=\mathrm{C}_{2} \mathrm{O}_{4}^{2-}$

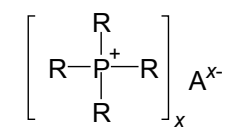

TBPE: $\mathrm{R}=n-\mathrm{Bu}, \mathrm{A}^{x-}=\mathrm{OAc}^{-}$

TBPA: $\mathrm{R}=n-\mathrm{Bu}, \mathrm{A}^{x-}=$ adipate

TBPM: $\mathrm{R}=n-\mathrm{Bu}, \mathrm{A}^{\mathrm{x}-}=$ malonate

图 4 离子型有机碱的结构 ${ }^{[6]}$

Figure 4 Structures of the organic ionic bases ${ }^{[6]}$

表 2 不同碱 $\left(0.01 \mathrm{~mol} \cdot \mathrm{L}^{-1}\right)$ 在有机溶剂中的电导率 $\left(\mu \mathrm{S} \cdot \mathrm{cm}^{-1}\right)\left(15{ }^{\circ} \mathrm{C}\right.$, $100 \mathrm{kPa})^{a}$

Table 2 Conductivity $\left(\mu \mathrm{S} \cdot \mathrm{cm}^{-1}\right)$ of various bases $\left(0.01 \mathrm{~mol} \cdot \mathrm{L}^{-1}\right)$ in organic solution $\left(15{ }^{\circ} \mathrm{C}, 100 \mathrm{kPa}\right)$

\begin{tabular}{ccc||ccc}
\hline Base & DMF & THF & Base & DMF & THF \\
\hline $\mathrm{Li}_{2} \mathrm{CO}_{3}$ & 2.63 & 0 & TBAP & 1237 & 21.70 \\
$\mathrm{Na}_{2} \mathrm{CO}_{3}$ & 2.75 & 0 & TBAC & 1229 & 19.00 \\
$\mathrm{~K}_{2} \mathrm{CO}_{3}$ & 14.00 & 0 & TBAHC & 716 & 8.76 \\
$\mathrm{Cs}_{2} \mathrm{CO}_{3}$ & 24.00 & 0.021 & TBAA & 1028 & 2.11 \\
$\mathrm{Na}_{3} \mathrm{PO}_{4}$ & 3.92 & 0 & TBAE & 721 & 1.28 \\
$\mathrm{~K}_{3} \mathrm{CO}_{3}$ & 13.10 & 0 & TBPA & 780 & 7.40 \\
TMAP & 185 & 1.01 & TBPE & 758 & 2.73 \\
TEAP & 740 & 2.88 & TBPM & 842 & 9.48 \\
\hline
\end{tabular}

${ }^{a}$ Conductivities of pure DMF and THF are 0.72 and $0 \mu \mathrm{S} \bullet \mathrm{cm}^{-1}$, respectively.

以 Heck 反应为例，使用 $\mathrm{Pd}(\mathrm{OAc})_{2}$ 作为催化剂、三 苯基膦作配体时, 通常需要加入碱来促使 $\operatorname{Pd}($ II) 转化为 活性 $\operatorname{Pd}(0)$ 物种(图 5) $)^{[7]}$. $\operatorname{Pd}(0)$ 相对 $\operatorname{Pd}(\mathrm{II})$ 来说是一个“裸” 的钯物种, 其反应活性更高. 在 $\operatorname{Pd}(\mathrm{II})$ 向 $\operatorname{Pd}(0)$ 转化的过 程中, 会生成质子和酸, 加入碱能中和质子, 促进这一 过程的进行.

另外，碱的加入也能中和体系里在还原消除过程中 产生的酸, 加速还原消除的进行, 促进活性催化物种的 再生 ${ }^{[8]}$.
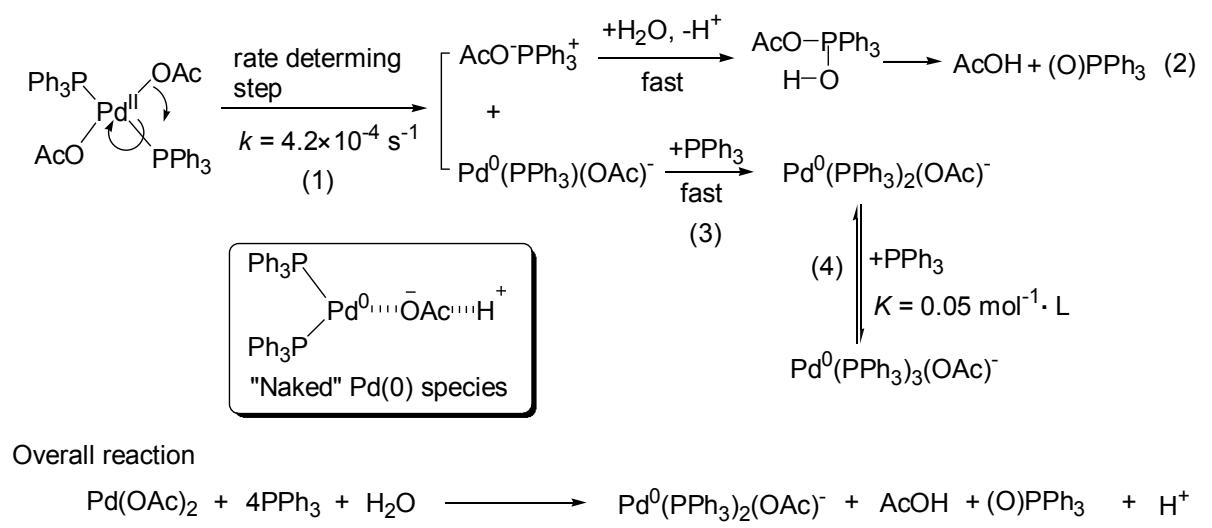

图 5 零价钯物种的生成 ${ }^{[7]}$

Figure 5 Generation of $\mathrm{Pd}(0)$ species $^{[7]}$ 


\section{3 阴离子的影响}

在很多反应中, 无机碱的阴离子部分可以作为配体 与催化剂中金属进行配位, 阻断中心金属的空配位点这 对催化剂的再生有很大的影响. 这样, 阴离子部分与中 心金属间的相互作用强弱和配位/解离速率都会影响到 反应的进程. 因此不同碱的选择会对反应的产率、速率 等产生影响.

Beller 等 ${ }^{[9]}$ 在 2001 年对图 6 所示的反应中离子的影 响进行了研究. 作者做了一系列实验, 向反应中添加各 种不同的阴阳离子, 以观察阴阳离子对反应的影响. 最 终的实验结果表明在这一反应中, 需要维持体系较低的 碱阴离子浓度才能正常进行, 同时, 不同阴离子对反应 结果的影响很大, 而阳离子对反应的影响基本只和溶解 度有关.

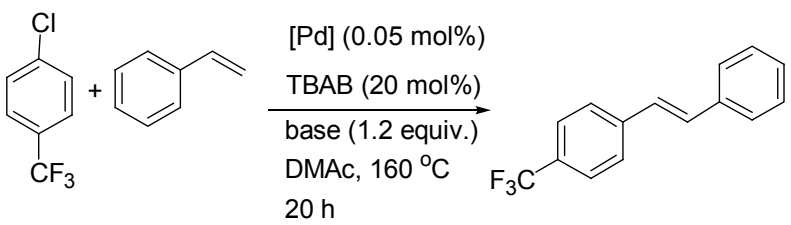

NaOAc: $64 \%$; KOAc: $61 \%$; $\mathrm{Na}_{2} \mathrm{CO}_{3}: 61 \%$; $\mathrm{CsCO}_{3}: 25 \%$ CaO: $67 \%$; NaOBu-t: $3 \%$; NEt $_{3}$ : $12 \%$

$\begin{array}{lcc:ccc}\text { Base: NaOAc } & & & \\ \text { Cation } & \mathrm{Anion} & \text { Yield/\% } & \text { Cation } & \text { Anion } & \text { Yield/\% } \\ \mathrm{Bu}_{4} \mathrm{~N}^{+} & \mathrm{Cl}^{-} & 21 & \mathrm{Li}^{+} & \mathrm{Cl}^{-} & 15 \\ \mathrm{Bu}_{4} \mathrm{~N}^{+} & \mathrm{Br}^{-} & 64 & \mathrm{Cs}^{+} & \mathrm{Cl}^{-} & 8 \\ \mathrm{Bu}_{4} \mathrm{~N}^{+} & \mathrm{I}^{-} & 35 & \mathrm{Bu}_{4} \mathrm{~N}^{+} & \mathrm{Cl}^{-} & 21 \\ \mathrm{Bu}_{4} \mathrm{~N}^{+} & \mathrm{SCN}^{-} & 1 & \mathrm{Li}^{+} & \mathrm{Br}^{-} & 54 \\ \mathrm{Bu}_{4} \mathrm{~N}^{+} & \mathrm{OAc}^{-} & 34 & \mathrm{Na}^{+} & \mathrm{Br}^{-} & 24 \\ \mathrm{Bu}_{4} \mathrm{~N}^{+} & \mathrm{OH}^{-} & 23 & \mathrm{~K}^{+} & \mathrm{Br}^{-} & 29 \\ \mathrm{Bu}_{4} \mathrm{~N}^{+} & \mathrm{NO}_{3}^{-} & 5 & \mathrm{Et}_{4} \mathrm{~N}^{+} & \mathrm{Br}^{-} & 31 \\ \mathrm{Bu}_{4} \mathrm{~N}^{+} & \mathrm{HSO}_{4}^{-} & 16 & \mathrm{Bu}_{4} \mathrm{~N}^{+} & \mathrm{Br}^{-} & 64 \\ \mathrm{Bu}_{4} \mathrm{~N}^{+} & \mathrm{BF}_{4}^{-} & 2 & \mathrm{Me}_{4} \mathrm{~N}^{+} & \mathrm{I}^{-} & 39 \\ \mathrm{Bu}_{4} \mathrm{~N}^{+} & \mathrm{OTs}^{-} & 4 & \mathrm{Et}_{4} \mathrm{~N}^{+} & \mathrm{I}^{-} & 33 \\ \mathrm{Bu}_{4} \mathrm{~N}^{+} & \mathrm{OTf}^{-} & 1 & \mathrm{Bu}_{4} \mathrm{~N}^{+} & \mathrm{I}^{-} & 35\end{array}$

图 6 阴阳离子效应对反应的影响 ${ }^{[9]}$

Figure 6 Anion effect and cation effect on the coupling ${ }^{[9]}$

不同碱的阴离子对反应的影响不仅体现在对产率 的影响上，同时也有可能对反应的选择性产生影响. Doucet 等 ${ }^{[10]}$ 报道过对于相同的底物, 当使用不同的碱 时，会得到不同的产物(图 7).

在这一反应中，碳酸铯能促进醚类产物的生成，而 其他金属的碳酸碱和乙酸碱只能促进 5 位的偶联反应发 生. 碳酸铯促进醚类产物的生成也被 Muzart ${ }^{[11]}$ 报道过, 但作者对碱的影响没有给出具体的解释.

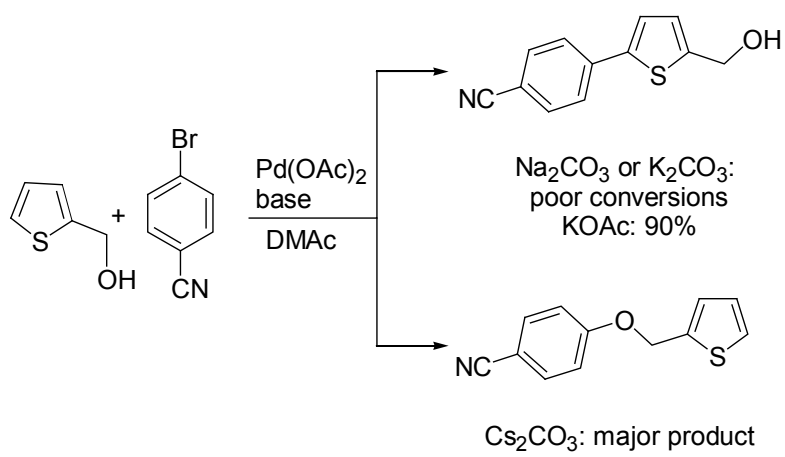

图 7 不同碱生成不同的产物 ${ }^{[10]}$

Figure 7 Formation of different products mediated by different base ${ }^{[10]}$

Doucet 等 ${ }^{[12]}$ 还报道了另一对类似的反应结果，也 是对同样的反应底物, 使用碳酸盐和乙酸盐会带来不同 的反应结果(图 8).<smiles>[R]NC(=O)c1[Y]([H])ccc1-c1ccccc1[R]</smiles>

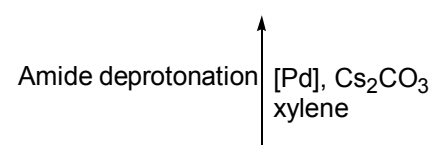
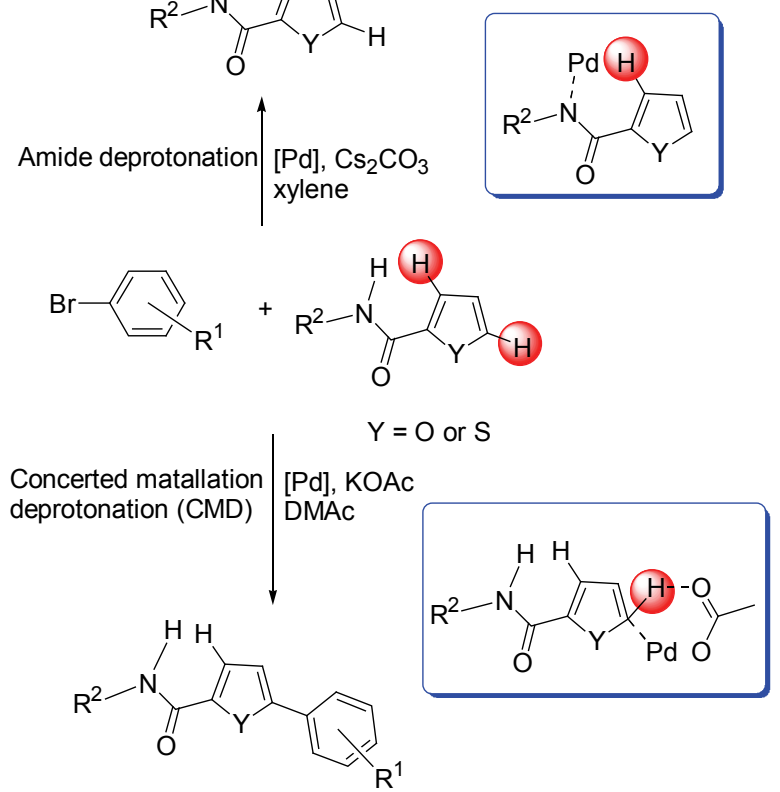

图 8 碱的性质和溶剂对芳基化反应的区域选择性的影响 ${ }^{[12]}$

Figure 8 Influence of the nature of the base and the solvent on the regioselectivity of the arylation reaction ${ }^{[12]}$

碱的阴离子部分可作为配体与催化剂中心金属配 位，进而对反应产生影响. 例如对于碳酸盐作用下的 $\mathrm{C}-\mathrm{H}$ 键活化反应，一般被认为是经历了一个碱协助的 协同的金属化/去质子化过程(图 9). 碳酸碱和乙酸碱对 图 7 这类反应的影响可以用 Fagnou 等 ${ }^{[13]}$ 提出的协同的 “金属化/去质子化” (concerted metalation/deprotonation, 简称 “CMD”，下同)机理来解释：碱与金属中心 Pd 配 位，同时对底物去质子化，从而活化 $\mathrm{C}-\mathrm{H}$ 键. 在这一 过程中, 碱与金属中心的配位作用是非常重要的. 除了 实验结果之外, 很多计算结果也表明在 $\mathrm{C}-\mathrm{H}$ 碱活化中, 使用碳酸(氢)碱和乙酸碱是比较有效的. 但具体使用哪 
一种, 则要看具体的反应体系. 例如在图 8 反应体系中, 虽然两种碱都能与中心金属配位，但反应并不都是 CMD 机理. 具体的反应结果也与体系碱性强弱有关系. 在很多环金属化反应中, 体系的 $\mathrm{pH}$ 值对反应的区域选 择性有显著影响 ${ }^{[14]}$.

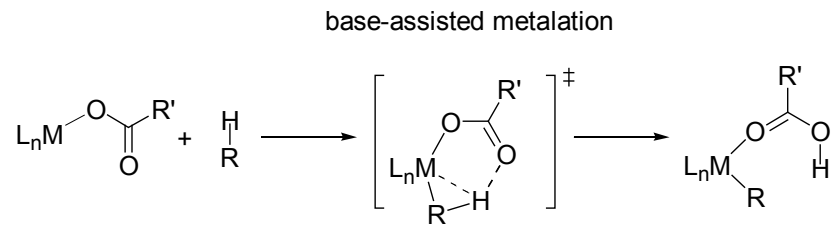

图 9 碱协助的协同的“金属化/去质子化”

Figure 9 Base assisted concerted metalation/deprotonation

Shaw 等在 70 年代早期发现, 向反应体系中添加醋 酸钠可以促进 $\mathrm{Ir}^{[15]}, \mathrm{Pt}^{[16]}, \mathrm{Pd}^{[17]}$ 配合物的环金属化反应. 例如在图 10 的反应中, 当没有碱加入时, 只能得到钯与 底物 7 的普通加合物 $\mathrm{PdCl}_{2}(7)_{2}$, 而在加入 1 equiv. 的碱 后, 则能顺利反应以 $84 \%$ 的收率得到钯杂五元环产物 $\mathbf{8}^{[17]}$. 在此基础之上, Sokolov 等 ${ }^{[18]}$ 在 1979 年报道了一例 光学活性氨基酸盐促进的胺(图 10 中化合物 7)导向的 $\mathrm{C}$ $-\mathrm{H}$ 键活化进而以较好的收率和 $e e$ 值得到光学活性产 物的反应. 作者提出在此反应中, 最重要的 $\mathrm{C}-\mathrm{H}$ 键金 属化过程经历了碱辅助的分子内协同的金属化/去质子 化机理, 其过渡态的结构如图 10 中 9 所示.

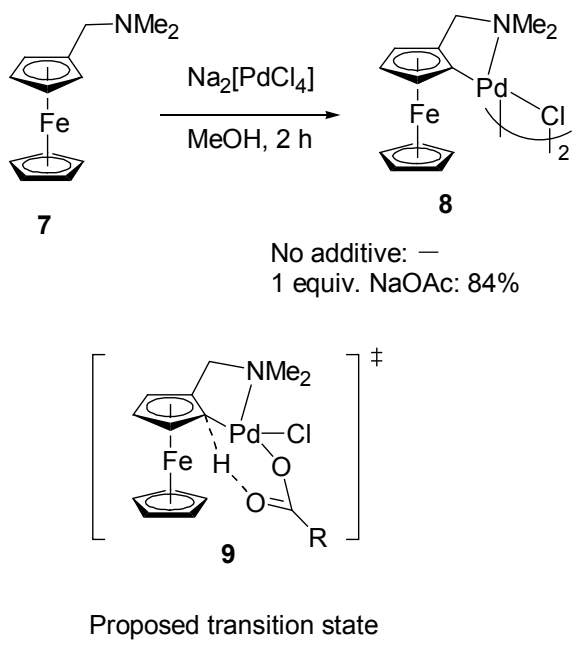

图 10 乙酸钠对环金属化反应的影响 ${ }^{[17,18]}$

Figure 10 Effect of $\mathrm{NaOAc}$ on the cyclopalladation ${ }^{[17,18]}$

过渡金属催化的反应中也有类似的过程. Fagnou 等 ${ }^{[13]}$ 为图 11 中的反应提出了 $\mathrm{CMD}$ 机理. 在碱的作用 下，氧化加成中间体进一步反应的过程可能有几种路 径, 图中所示的 Path 1 和 Path 2 是氧化加成后的产物分 别与两种碱 $\left(\mathrm{Br}^{-}\right.$和 $\left.\mathrm{CO}_{3}^{2-}\right)$ 作用的机理. 这个机理是由 Echavarren 和 Maseras 等 ${ }^{[19]}$ 在一个分子内芳基化反应中 提出的. 对于碱参与的路径, Fagnou 等对不同碱的篗氢 反应进行了研究, 提出了四条路径, 都与协同的金属化 过程和擢氢反应联系十分紧密. 它们之间的不同在于活
性催化物种和篗氢的碱的不同. 在 Path 1 中, 活性催化 物种是 $\left(\mathrm{PR}_{3}\right) \mathrm{ArPd}-\mathrm{Br}$ 配合物而擢氢的是 $\mathrm{Br}$ 配体．在 Path 2 中, $\mathrm{Pd}$ 上的 $\mathrm{Br}$ 被 $\mathrm{HCO}_{3}^{-}$离子取代, 此时活性催化物种 是 $\left(\mathrm{PR}_{3}\right) \mathrm{ArPd}-\mathrm{HCO}_{3}$. 在 Path 3 中活性催化物种是 $\left(\mathrm{PR}_{3}\right) \mathrm{ArPd}-\mathrm{Br}$, 这一活性物种与含氟底物之间有的弱的 相互作用，此时反应通过游离的 $\mathrm{HCO}_{3}^{-}$离子进行分子 间的擢氢反应. 而事实上因为 $\mathrm{HCO}_{3}^{-}$离子在进行睅氢 的过程前是与 $\mathrm{Pd}$ 中心配位的，因此这个路径并不合理. 这也从侧面表明，反应中的活性催化物种可能不是一个 三配位的钯物种而是一个同时与 $\mathrm{Br}$ 和 $\mathrm{HCO}_{3}$ 配位的四 配位钯物种, 如 Path 4 所示. 进一步的计算结果表明, Path 2 中 $\mathrm{HCO}_{3}^{-}$离子的擢氢过程反应能垒最低, 也与实 验数据相符. 同时, Fagnou 等 ${ }^{[20]}$ 也指出, 碱性的强弱和 反应体系中离子的浓度(离子浓度不够时, 碱协助的反 应过程无法进行)都对反应进程有影响.

对于碱协助的擢氢机理, Echavarren 等 ${ }^{[21]}$ 也做了研 究, 例如在图 12 的反应中, 当使用的碱为 $\mathrm{K}_{2} \mathrm{CO}_{3}$ 时, 可 以得到令人满意的反应结果, 而使用有机碱 $\mathrm{DBU}$ 和三 乙胺时, 则反应不能发生. 这个反应的区域选择性与所 使用的碱有密切关系. 若使用较强的碱如 $\mathrm{KOBu}-t$, 则 只能得到脱溴的产物. 若向反应体系中加入 $\mathrm{PivOH}$, 则 还能进一步地提高反应收率.

对于该反应作者提出了三种可能的路径如图 13 所 示. 从氧化加成得到的中间体出发, 质子可以转移到 $\mathrm{Br}$ 上(非碱协助的机理)或碱上(碱协助的机理). 对于碱协 助的反应机理，有可能有两条路径：(i)通过分子内与 $\mathrm{Pd}$ 配位的碳酸根进行协同的去质子化过程得到产物; (ii)通 过体系中游离的碳酸根对苯环拔氢, 去质子化得到最终 产物. 计算结果表明, 非碱协助的反应路径比碱协助的 反应路径能垒高 $20 \mathrm{kcal} / \mathrm{mol}$ 以上. 由此证明，这个反应 中比较可能的机理是碱协助的反应过程 ${ }^{[21,22]}$.

Baudoin 等 ${ }^{[23]}$ 在此方面也做了一些研究(图 14). 作 者对碱协助的环金属化反应进行了计算研究. 计算结果 表明，与金属中心配位的碱对 $\mathrm{C}-\mathrm{H}$ 键活化的过程影响 显著. 而同时实验结果也表明，在这一反应中 $\mathrm{K}_{2} \mathrm{CO}_{3}$ 是 反应效果最好的碱, $\mathrm{KHCO}_{3}$ 次之, 而 $\mathrm{KOAc}$ 基本不能促 进该反应的进行. 尽管在很多实验过程中发现碳酸盐的 反应效果较好, 很多计算研究使用的模型依然是碳酸氢 盐和醋酸盐. 接着作者对这一反应的过程进行了计算研 究. 对于 $\mathrm{OAc}^{-}$活化 $\mathrm{C}-\mathrm{H}$ 的反应过程, 计算得到的能垒 是 $138 \mathrm{~kJ} / \mathrm{mol}, \mathrm{HCO}_{3}^{-}$活化的反应进程, 能垒是 143.5 $\mathrm{kJ} / \mathrm{mol}$, 而对于 $\mathrm{CO}_{3}^{2-}$ 活化的进程, 能垒是 $187.9 \mathrm{~kJ} / \mathrm{mol}$. 这一计算结果与实际的反应结果恰好是完全相反的. 计 算结果同时表明，碱取代 $\mathrm{Br}^{-}$与金属配位是一个放热过 程, 有较强的热力学驱动力. 使用不同的碱作为模型计 算出的反应活化能大小相似, 但实验证明 $\mathrm{CO}_{3}^{2-}$ 的反应 能垒相对较低. 而反应中碱的作用之一是与中心金属配 位进而进行篗氢的过程. 


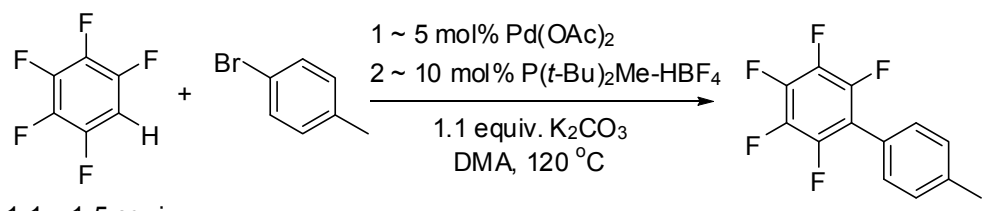

$1.1 \sim 1.5$ equiv.

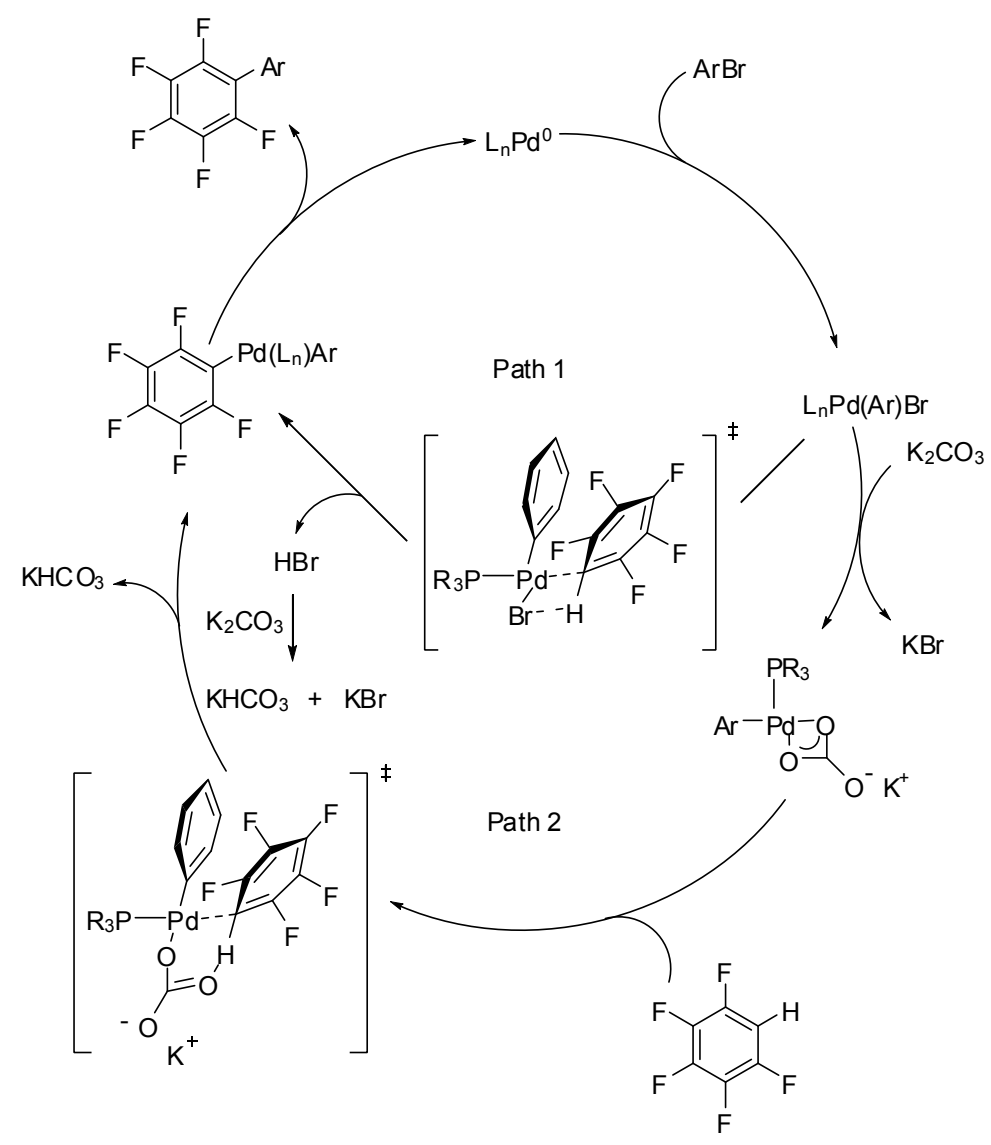

Path 1: concerted metal lation and proton abstraction by $\mathrm{Br}$ to eliminate $\mathrm{HBr}$-mechanism Path 2: concerted metallation and proton abstraction by coordinated bicarbonate-mechanism<smiles>[R7][Pb](Br)(c1ccccc1)c1ccccc1</smiles>

Path 3: concerted metallation and proton abstraction by a free bicarbonate-mechanism<smiles>[R6][PbH](OC([O-])O)c1ccccc1</smiles>

Path 4: exchange reaction of carbonate ligand with flouroarene-mechanism

图 11 五氟苯的芳基化反应及不同的碱协助的反应机理 ${ }^{[13]}$

Figure 11 Direct arylation of perfluorobenzenes and several pathways for base assisted mechanism ${ }^{[13]}$ 
<smiles>[X]c1ccc(C(Cc2ccccc2Br)c2ccccc2)cc1</smiles>

10a: $\mathrm{X}=\mathrm{Cl}$

10b: $X=F$
(1) $\mathrm{Pd}(\mathrm{OAc})_{2}$, ligand base, solvent, heating

(2) $D D Q$, toluene, heating

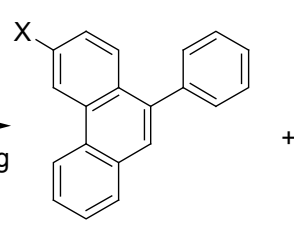

11a: $\mathrm{X}=\mathrm{Cl}$ 11b: $X=F$

Base

$\mathrm{K}_{2} \mathrm{CO}_{3}$

DBU

$\mathrm{Et}_{3} \mathrm{~N}$

$\mathrm{K}_{2} \mathrm{CO}_{3} / \mathrm{PivOH}$<smiles>[X]c1ccc(-c2cc3ccccc3c3ccccc23)cc1</smiles>

12a: $\mathrm{X}=\mathrm{Cl}$

12b: $X=F$

$11 / 12$ ratio

2:1

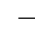

$1.7: 1$

图 12 钯催化的分子内芳基化反应 ${ }^{[21]}$

Figure 12 Palladium-catalyzed intramolecular arylation ${ }^{[21]}$

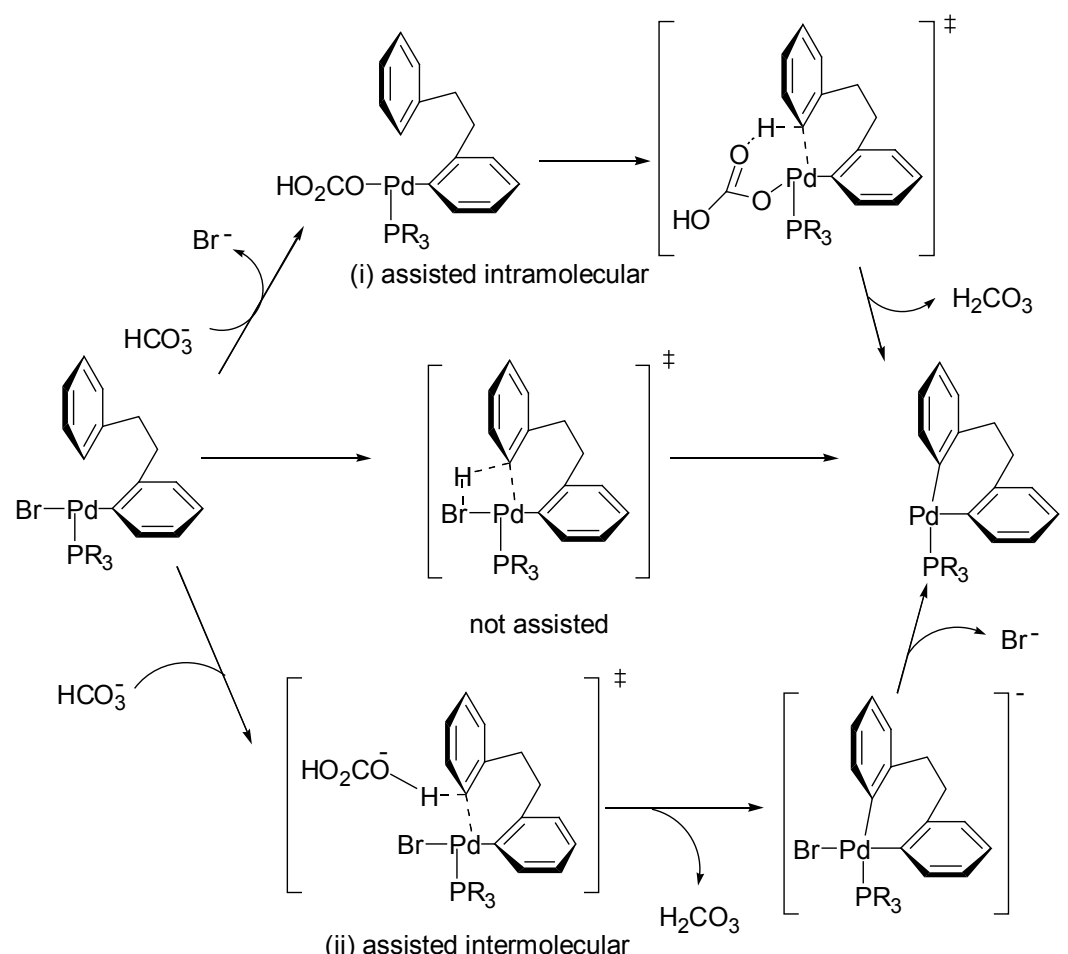

图 13 分子内芳基化反应中 $\mathrm{C}-\mathrm{H}$ 键活化生成钯杂环的几种路径 ${ }^{[21,22]}$

Figure 13 Pathways for $\mathrm{C}-\mathrm{H}$ bond palladation in intramolecular direct arylations ${ }^{[21,22]}$<smiles>CC(=O)C(C)(C)c1ccccc1Br</smiles>

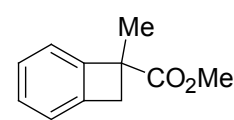

$150{ }^{\circ} \mathrm{C}, 1.5 \mathrm{~h}$

图 14 钯催化的甲基中的 $\mathrm{C}-\mathrm{H}$ 键活化 ${ }^{[23]}$

Figure 14 Palladium-catalyzed $\mathrm{C}-\mathrm{H}$ activation of methyl groups ${ }^{[23]}$

另外, 在很多 $\mathrm{C}-\mathrm{H}$ 活化的反应中, 加入 $\mathrm{PivOH}$ 能 促进反应的进行 ${ }^{[21,24,25]}$. 这是因为在大多数反应中, 无 机碱在有机溶剂中并不能很好地溶解. $\mathrm{PivOH}$ 作为一个

可溶性的质子转移试剂能将底物和钯催化剂上的质子 转移到碱上, 从而促进反应的进行. 而同时, $\mathrm{PivO}^{-}$可作 为配体与中心金属配位, 发生与 $\mathrm{CO}_{3}^{2-}$ 活化 $\mathrm{C}-\mathrm{H}$ 键类 似的反应过程.

含氟化合物，例如 CsF、四丁基氟化铵(TBAF)等也 是过渡金属催化的反应中常用的碱. 碱金属氟化物只在 少数溶剂中有明显的溶解性. $\mathrm{KF}, \mathrm{RbF}$ 和 $\mathrm{CsF}$ 在一些羧

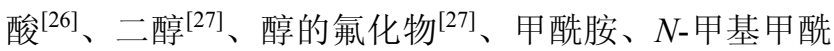
胺 $^{[28]} \mathrm{HF}$ 和水中可溶. 但这些溶剂中, 没有一个能适用 于所有氟离子源. 目前关于碱金属氟化物促进的反应的 报道多数集中在 $\mathrm{KF}$ 和 $\mathrm{CsF}$ 上. 而在非质子溶剂中使用 $\mathrm{KF}$ 或 $\mathrm{CsF}$ 反应时，一般认为有相当数量的反应发生在 
未溶解的氟化物的表面, 同时反应需要始终进行充分摚 拌才能顺利进行 ${ }^{[29]}$. 在质子溶剂中, 氟离子可以被其自 身与溶剂间的强氢键相互作用所稳定. 这个氢键作用也 会降低氟离子的亲核性同时提高溶剂的亲核性. 当碱金 属氟化物作为碱 ${ }^{[30]}$ 或亲核试剂参与反应时, 向反应体 系中加入冠醚(例如向 KF-benzen 或 KF-MeCN 体系中加 入 18-冠-6)后会极大地提升碱的溶解度(至少 10 倍), 进 而对反应结果有极大的影响 ${ }^{[27]}$.

在过渡金属催化的反应中, 氟化物常被当作碱用于 含硅化合物的偶联反应中 ${ }^{[31,32]}$. 例如在 Hiyama 偶联反 应中, $\mathrm{Si}-\mathrm{C}$ 键的极化是至关重要的一步, 而这一步需
要氟离子或其它碱的参与，与硅配位形成一个五配位的 硅中间体，活化有机硅中低极化的 $\mathrm{Si}-\mathrm{R}$ 键，进而发生 后续反应(图 15).

此外，在 Suzuki 反应中加入氟离子会与芳基硼酸形 成氟嗍酸盐负离子，可以促进硼酸盐中间体与钯中心的 反应. 因此, $\mathrm{TBAF}, \mathrm{CsF}, \mathrm{KF}$ 等化合物都可以使该反应 速率加快, 甚至可以代替反应中使用的其他碱.

由于阳离子的不同，不同的氟化物会导致反应结果 有差异. 因此, 可以通过对氟化物的选择来调控反应的 选择性. 例如 Hiyama 等 ${ }^{[32]}$ 报道(图 16), 当使用三(二甲 氨基甲基)锍二氟三甲基氨酸硅酸盐(TASF)时，由于阳
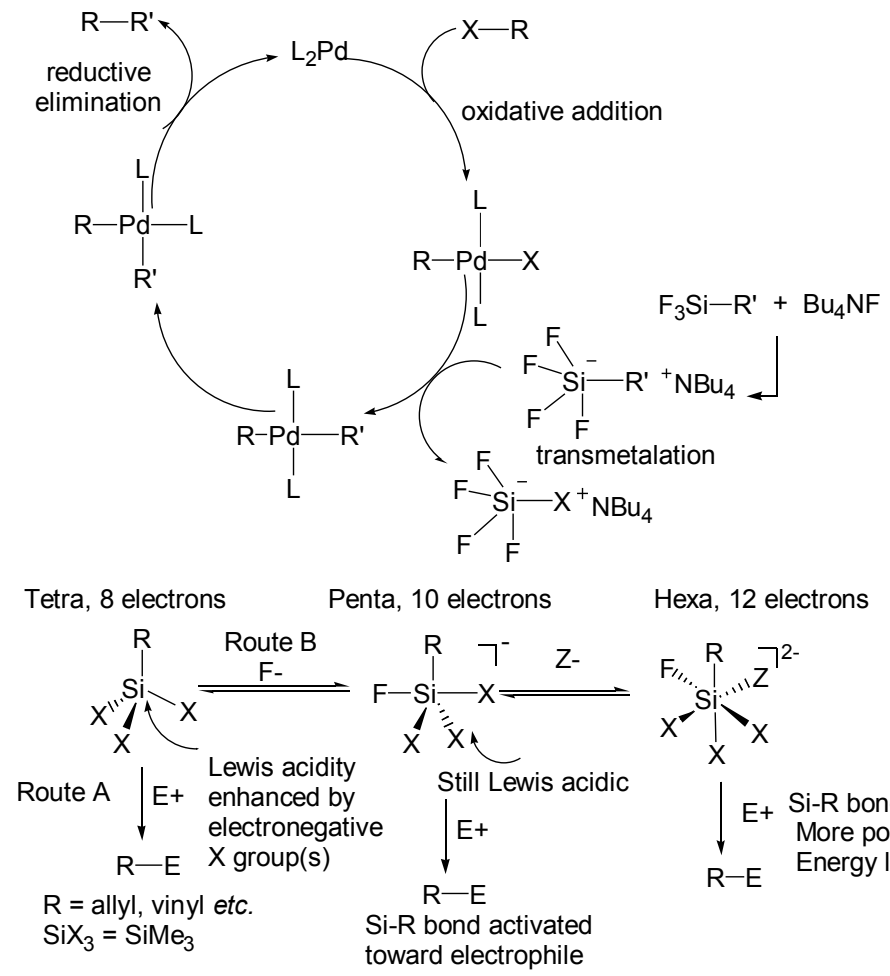

Hexa, 12 electrons

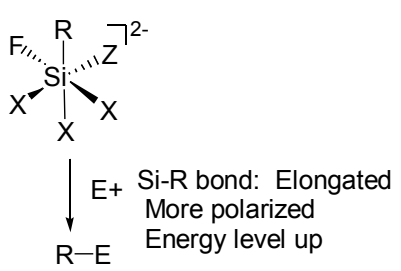

图 15 Hiyama 偶联反应机理及氟离子的作用

Figure 15 Mechanism for Hiyama coupling and role of fluoride ion<smiles>CC/C=C/C(C)c1ccc2ccccc2c1</smiles>

$(S)-(E) 64 \%$

24:1, E/Z

$82: 18, S / R$

$91 \%$ es

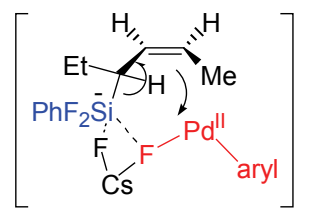

syn $\mathrm{S}_{\mathrm{E}}{ }^{\prime}$<smiles>CC=CC(C)c1ccc2ccccc2c1</smiles>

$(S)-(E) 50 \%$

$42: 1, E / Z$

$24: 76, S / R$

$74 \%$ es

图 16 氟离子源和溶剂对芳基化反应的对映选择性的影响 ${ }^{[32]}$

Figure 16 Influence of the fluoride source and the solvent on the enantioselectivity of the arylation reaction ${ }^{[32]}$ 
离子位阻较大, 此时反应中钯物种会从上方与双键作 用, 得到 anti- $\mathrm{S}_{\mathrm{E}^{\prime}}$ 产物; 而当使用 $\mathrm{CsF}$ 时, 此时阳离子较 小, Pd-F-Si 间相对较强的相互作用会使钯物种从双键下 方作用, 得到 $s y n-\mathrm{S}_{\mathrm{E}^{\prime}}$ 产物.

\section{4 阳离子的影响}

阳离子对反应的影响也很多. Suzuki 偶联反应在没 有碱的参与时是很难发生的. 在该反应中, 碱最重要的 作用是促进转金属反应的进行(图 17) ${ }^{[33]}$. 反应中碱的影 响不仅取决于碱(负离子)的强弱, 而且也与阳离子的性 质有关. 阳离子对反应的影响是多方面的. 其离子大 小、软硬度、Lewis 酸性、在溶液中的聚集度 ${ }^{[34]}$ 等都有 可能对反应结果造成影响. 例如在 Suzuki 偶联反应中阳 离子如果太小不利于生成中间的过渡态 $y \operatorname{lide}(\mathrm{Pd})$ 中间 体. 通常来说, 阳离子大的碱, 如 $\mathrm{Ba}, \mathrm{Cs}$ 等会加速反应, 阳离子太小而被屏蔽, 反应的速率和效率将显著下降. Suzuki 反应中, 最常用的是碳酸钠、碳酸铯、醋酸钾、 磷酸钾等. 碱金属碳酸盐中, 反应活性顺序为: $\mathrm{Cs}_{2} \mathrm{CO}_{3}>$ $\mathrm{K}_{2} \mathrm{CO}_{3}>\mathrm{Na}_{2} \mathrm{CO}_{3}>\mathrm{Li}_{2} \mathrm{CO}_{3}$.

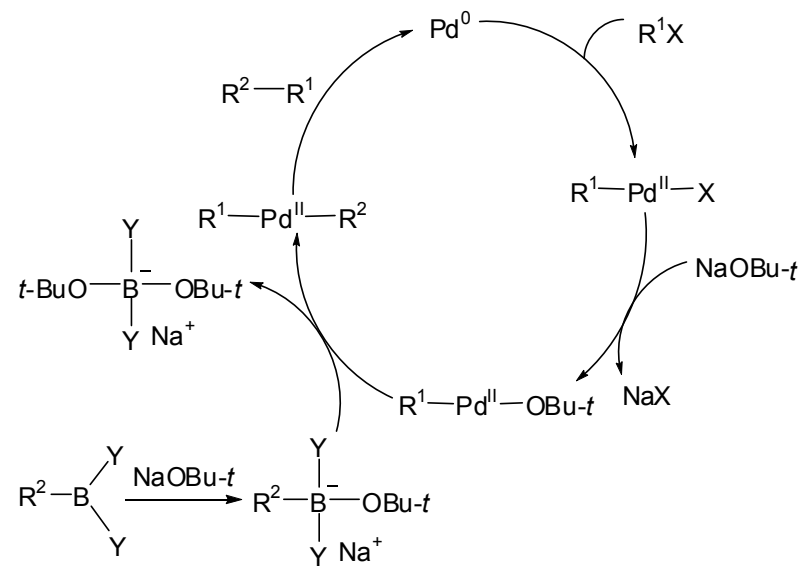

图 17 Suzuki 偶联反应机理

Figure 17 Mechanism for Suzuki coupling

对于相同阴离子的碱, 不同的阳离子也可能对反应 结果带来不同影响 ${ }^{[35]}$. 阳离子可能影响反应的活性中 间体. Shibasaki 等 ${ }^{[36]}$ 在 2009 年报道了图 18 中的反应. 叔丁醇锂、钠、钾都能促进这一反应的进行. 但反应的 收率和 $e e$ 值对应的碱都是 $\mathrm{LiOBu}-t>\mathrm{NaOBu}-t>\mathrm{KOBu}-t$. 作者推测在这一反应中, 首先是叔丁醇阴离子与一价铜 盐上的阴离子进行交换, 得到活性的催化物种 $\mathrm{CuOBu}-t$, 接着进行随后的反应. $\mathrm{Li}, \mathrm{Na}, \mathrm{K}$ 三种碱金属叔丁醇盐的 区别只是阳离子的不同, 因此作者推测该阳离子应该在 反应中起了作用. 于是作者向反应中加入不含锂离子的 铜催化剂 CuOBu- $t$ 进行反应, 发现这一反应仍然能发 生，但是反应收率只有 $31 \%$ 同时 $e e$ 值为 $75 \%$. 作为对比 实验, 向这一反应体系中加入锂盐 $\mathrm{LiPF}_{6}$ 后进行反应, 则反应顺利进行, 以 $80 \%$ 的产率和 $87 \%$ ee 值得到预期 产物. 这也证明了锂离子在这一反应过程中是十分重要
的 $^{[37]}$. 而三种碱金属盐得到的不同反应结果，可能与三 种金属的离子半径不同有关系，也可能与碱金属离子与 铜物种形成簇合物有关系。同时，由于很多铜盐催化剂 对反应体系的碱性十分敏感，在强碱性环境中铜盐相对 较容易聚集从而使其反应活性受到影响. 因此该反应中 所使用碱的碱性强弱也有可能是造成依次使用叔丁醇 锂、钠、钾时反应收率和 $e e$ 值会依次下降的原因之一.

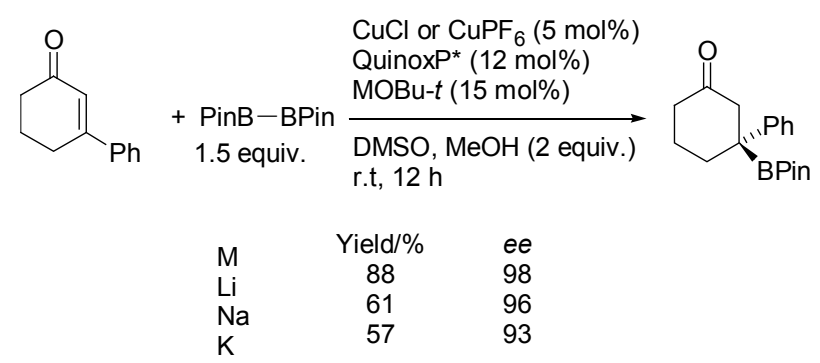

图 18 通过不对称催化合成手性有机硼酯的反应中的阳离子效应 ${ }^{[36]}$ Figure 18 Cation effect in catalytic asymmetric synthesis of chiral tertiary organoboronic esters ${ }^{[36]}$

Fagnou 等 ${ }^{[38]} 2006$ 年报道的图 19 的反应中, 阴阳离 子对反应的产率和选择性都有显著影响. 碳酸碱的反应 收率比乙酸碱的反应收率高. 而对于阴离子相同的碱, 则 $\mathrm{K}$ 离子碱的反应转化率比 $\mathrm{Na}$ 离子的高, 选择性也较 好. 阳离子相同的碱反应得到的选择性类似. 而有机碱 三乙胺则几乎不能促进这一反应的进行. 为了了解这一 反应中的碱的作用, 作者比较了碳酸钠、钾、铯在该反 应条件下的溶解度。实验证明在该反应的反应条件下, 三种碱都是几乎不溶的, 因此作者认为这些碱在 DMA 中的溶解度差异不是导致反应结果不同的原因.<smiles>Clc1ccccc1COc1ccccc1</smiles>
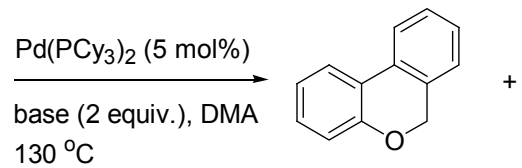

13<smiles>c1ccc(COc2ccccc2)cc1</smiles>

Conversion/\%
11
100
25
81
41
3

Ratio 13:14 20:1 $>99: 1$ 15:1 $>99: 1$ 28:1 2:1
图 19 芳基氯化物的直接芳基化反应 ${ }^{[38]}$

Figure 19 Direct arylation of aryl chlorides ${ }^{[38]}$

在 Doucet 和 Dixneuf 等 ${ }^{[39]} 2011$ 年报道的反应中 (图 20), 作者讨论了 $\mathrm{Na}, \mathrm{K}, \mathrm{Cs}$ 的醋酸盐对反应产率的影 响 $(\mathrm{Na}<\mathrm{K})$ ，作者对于产率不同的解释是这个反应经历 的是 CMD 路径, 使用 $\mathrm{KOAc}$ 和 CsOAc 时乙酸根向 $\operatorname{Pd}(\mathrm{II})$ 转移的比较快, 能较快地生成随后㩖氢反应中所 需的活性钯物种. 但这不能解释为什么碳酸盐不能很好 地促进这一反应的进行. 


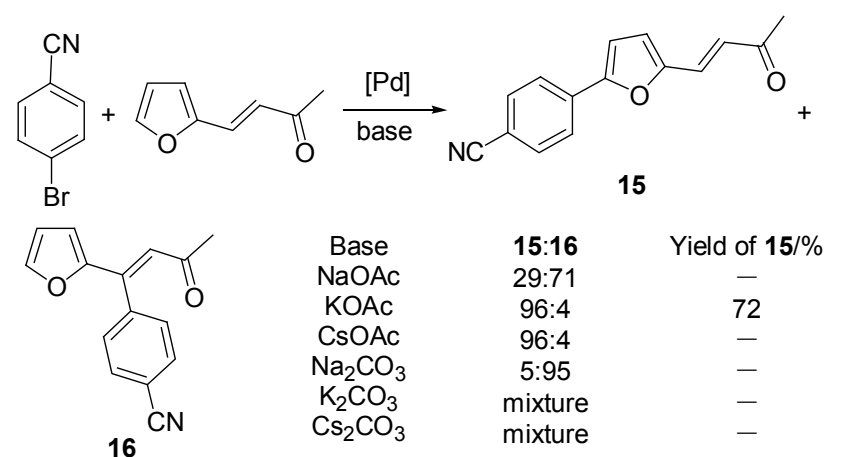

图 20 呋喃衍生物的选择性直接芳基化反应 ${ }^{[39]}$

Figure 20 Selective direct arylation of furans ${ }^{[39]}$

Hayashi 等 ${ }^{[40]}$ 在 2010 年报道的反应中, 也有提及 $\mathrm{MOBu}-t(\mathrm{M}=\mathrm{Li}, \mathrm{Na}, \mathrm{K})$ 中阳离子对反应的影响(图 21). 在这一反应中, 使用 $\mathrm{MOBu}-t(\mathrm{M}=\mathrm{Li}, \mathrm{Na}, \mathrm{K})$ 作碱时, 反 应速率随着离子半径的增大而加快. 与 $\mathrm{NaOBu}-t$ 促进的 反应相比, 使用 $\mathrm{KOBu}-t$ 时, 虽然反应速率较快, 但反应 收率略低. 这些实验结果与该反应中涉及的单电子转移 (single electron transfer, 简称 SET) 机理相符, 即 $t$ - $\mathrm{BuO}^{-}$ 是一个单电子供体, 与之相连的金属有较高的电子密度 时, 表现出较高的反应性.

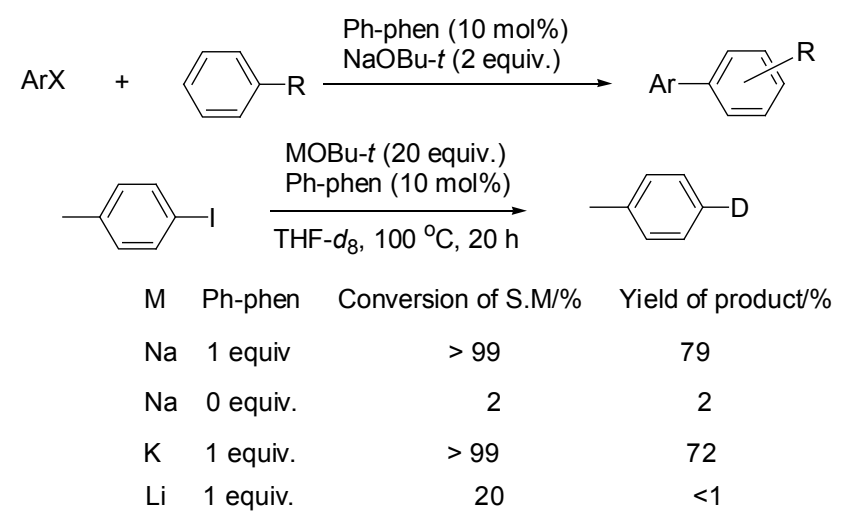

图 21 芳烃与甾代芳烃的芳基化反应中的阳离子效应 ${ }^{[40]}$

Figure 21 Cation effect in arylation of arene and aryl halides ${ }^{[40]}$

目前也有一些关于离子与溶剂间的作用力或与阴 离子间作用力的强弱对反应影响的文献报道 ${ }^{[41,42]}$. 其 中, 离子与溶剂间的作用力的影响在碱促进的氢转移反 应中表现显著.

Bäckvall 等 ${ }^{[43]}$ 在 1991 年报道了图 22 的反应. 环己 酮在钓催化剂和氢氧化钠的作用下, 在 2-丙醇中可以转 化为环己醇. 实验结果表明, 在该氢转移过程中, 过渡 金属催化剂和碱的参与都是不可缺少的. 若只向反应溶 液中加入 $\mathrm{RuCl}_{2}\left(\mathrm{PPh}_{3}\right)_{3}$ 催化剂并升温至 $82{ }^{\circ} \mathrm{C}$ 反应时, 并不能监测到相应产物的生成. 而在此基础上, 再向反 应混合物中加入 $2.4 \mathrm{~mol} \%$ 的 $\mathrm{NaOH}$ 后则引发了氢迁移 反应的发生, $1 \mathrm{~h}$ 后 $89 \%$ 的环己酮转化为了环己醇, 相当 于转化频率是 $890 \mathrm{~h}^{-1}$. 有意思的是, 在这个反应中, 反 应的前 $15 \mathrm{~min}$ 中, 反应的转化频率是 $1800 \mathrm{~h}^{-1}$. 这个结
果比在中性环境中的反应有显著的提高. 而若将反应中 生成的丙酮及时地从体系中除去, 则环己酮能完全转化 为环己醇.

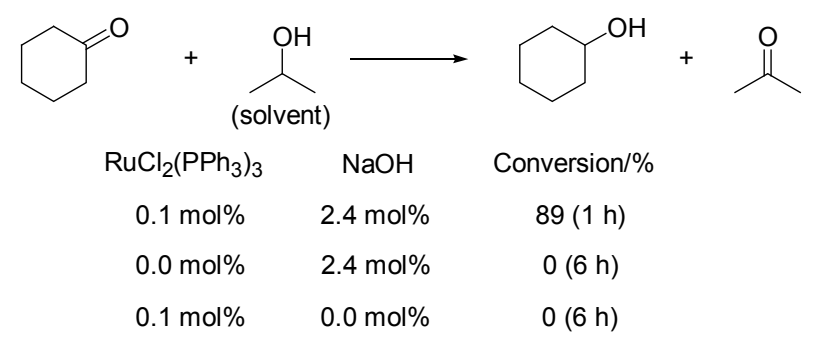

图 22 钉催化剂和氢氧化钠促进的氢转移反应 ${ }^{[43]}$

Figure $22 \mathrm{Ru}$ catalyst and $\mathrm{NaOH}$ promoted $\mathrm{H}$-transfer reaction ${ }^{[43]}$

该钉/氢氧化钠促进的氢转移过程的机理与 ColeHamilton 等 ${ }^{[44]}$ 描述的机理类似，即：(i)异丙醇阴离子首 先进攻钓配合物，生成一个阴离子活性物种; (ii)烷氧基 配体上的 $\beta$-氢消除生成钓一氢阴离子配合物和丙酮; (iii) 阴离子物种的快速质子化进而生成二氢钓物种 $\mathrm{RuH}_{2}(\mathrm{PPh})_{3} ;$; (iv)二氢钉物种还原环已酮得到产物环己 醇。在此反应中, 碱的作用是促进活性催化物种 $\mathrm{RuH}_{2}(\mathrm{PPh})_{3}$ 的形成.

对该类氢转移反应进一步研究发现过渡金属催化 剂并不是必须的, 仅使用碱也能促使该氢转移过程的进 行, 但此时需要大量的碱 ${ }^{[45,46]}$. Varma 等 ${ }^{[45 a]}$ 在 2009 年报 道, 仅使用 $\mathrm{KOH}$ 也可以在 2-丙醇中还原芳香醛; Ouali 等 ${ }^{[45 b]}$ 也在同一年报道了 $\mathrm{NaOH}$ 是一些芳香族和脂肪族 酮类化合物的活性氢转移催化剂. 其他多种碱如 $\mathrm{K}_{3} \mathrm{PO}_{4}$, $\mathrm{NaOBu}-t, \mathrm{KOBu}-t$ 等也被多个小组证实是促进很多酮类 化合物发生氢转反应的试剂 ${ }^{[46]}$. 目前使用催化量碱 ${ }^{[45]}$ 促进氢转移反应的报道很少.

在对这些反应研究的过程中, Ouali 等 ${ }^{[56 b]}$ 发现, 在 使用阴离子相同的碱时, 不同的金属阳离子会对反应产 率产生影响(图 23). 含钠的碱总是得到最高的产率，而 使用含锂的碱则产率较低.

这个反应结果可以通过图 24 中的机理来解释: 首 先是 2-丙醇在碱的作用下去质子化(步骤 1)得到 2-醇金 属盐物种，该物种可以通过金属离子与氧原子配位来活 化酮形成一个六元环中间体. 接着氢转移到金属盐物种 上同时丙酮解离出来(步骤 3). 这个醇盐与 2-丙醇反应 即得到预期的产物醇，同时再生出 2-醇金属盐物种(催 化剂)(步骤 4). 在这个反应的第一步中, 即去质子化过 程中, 并没有观察到该过程与所使用的碱的碱性强弱有 直接关联, 因此这个反应的决速步应该在步骤 2 4 中. 而步骤 2 4 可以看作是一个 Lewis 酸(金属离子 $\mathrm{M}$ )与 Lewis 碱(酮或醇盐)的配位活化和解离的过程. 这个过 程的速率是由 $\mathrm{M}-\mathrm{O}$ 键的强弱来控制的 ${ }^{[47 a]}$. 步骤 2 中, 相对较硬的酸更容易配位 $(\mathrm{Li}>\mathrm{Na}>\mathrm{K})$, 而在解离的过 程中, 软酸离子更倾向于解离 $(\mathrm{K}>\mathrm{Na}>\mathrm{Li})$. 钠离子在 


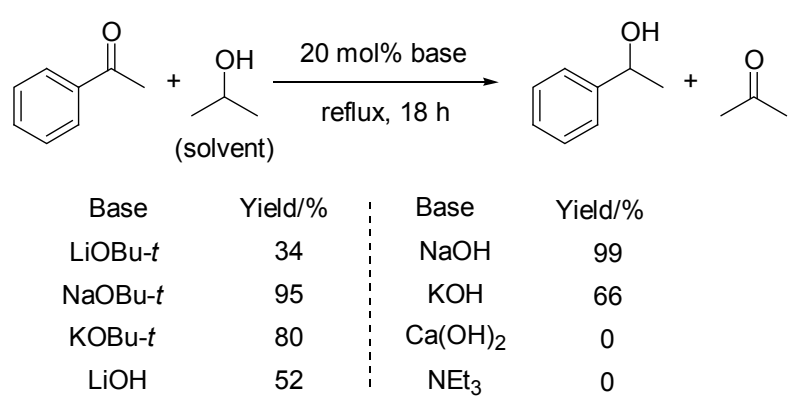

图 23 氢转移反应中阳离子的影响 ${ }^{[45 b]}$

Figure 23 Cation effect in $\mathrm{H}$-transfer reaction ${ }^{[45 b]}$

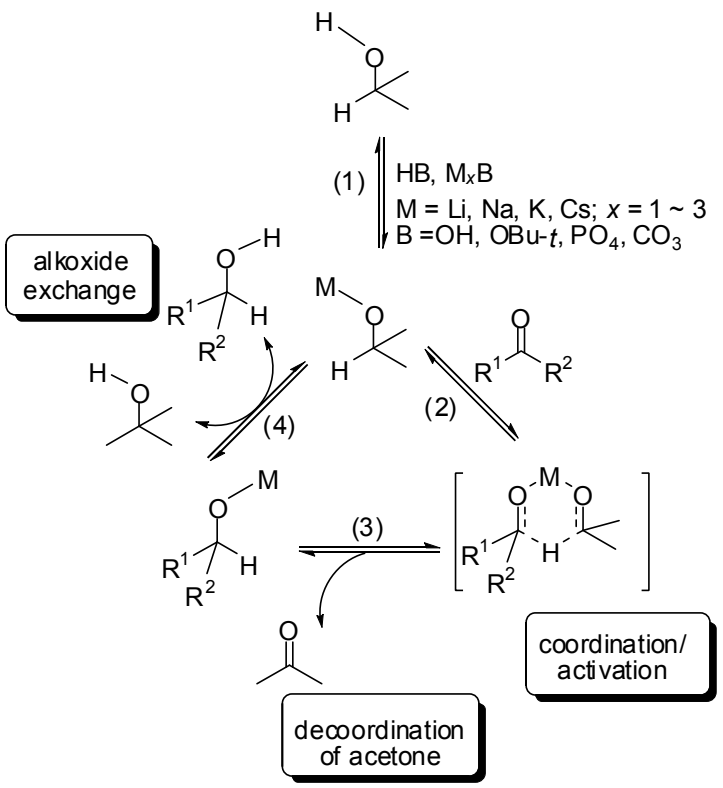

图 24 碱促进的氢转移反应机理 ${ }^{[45 \mathrm{~b}]}$

Figure 24 Mechanism for base promoted $\mathrm{H}$-transfer reaction ${ }^{[45 b]}$

这两个过程中反应活性相对平衡, 因此最终得到的反应 结果最理想 ${ }^{[48]}$. 从这一过程中可以看出, 无机碱的中心 金属与溶剂或底物的配位作用强弱对反应结果有十分 重要的影响.

在对亚胺 $\alpha$-碳负离子的钯催化烯丙基取代反应研 究中, 侯雪龙、吴云东等讨论了 $\mathrm{Li}^{+}, \mathrm{K}^{+}$阳离子的 Lewis 酸性和“软硬”度对该反应的化学和区域选择性影响 ${ }^{[47 b]}$. 在对亲核氟烷基化反应的研究中, 胡金波等讨论了 $\mathrm{M}-$ $\mathrm{O}$ 键的强弱对反应立体选择性的影响 ${ }^{[47 \mathrm{c}]}$, 并发现在某 些情况下, 碱中金属阳离子只有与氟原子取代一起发挥 作用, 才能够促进碳一碳键的高效形成 ${ }^{[47 \mathrm{~d}]}$.

\section{5 有机碱与无机碱的比较}

在过渡金属催化的反应中, 碱的选用范围是比较广 的. 有机碱和无机碱都被广泛地应用于各类反应中. 但 这两种碱有着很不一样的性质, 导致在同一反应中, 使 用无机碱和有机碱的反应结果相差很大.

例如在 DMF 中, $\mathrm{K}_{2} \mathrm{CO}_{3}$ 和 DBU 的碱性是几乎相同
的, 但它们的反应活性却有很大的差别 ${ }^{[19,21,49]}$. Echavarren 等 ${ }^{[19,21]}$ 在 2007 年报道的图 12 中的反应中, 当使 用 $\mathrm{K}_{2} \mathrm{CO}_{3}$ 时, 反应能够很顺利的进行, 以较好的收率得 到相应产物; 当使用 DBU 时, 则不能观察到相应产物 的生成. 这可能是由于 DBU 较大的空阻使得反应不能 顺利进行.

有机碱与无机碱的溶解性差异也会造成反应结果 不同. 对于同一反应, 分别使用相同当量有机碱和无机 碱进行反应时，由于两者在有机溶剂中的溶解度差异较 大, 会使得实际反应溶液的碱性不同 ${ }^{[50]}$, 从而使反应结 果相去甚远.

\section{6 结束语}

碱在有机反应中的作用很多, 影响反应的因素也很 多. 除了本文中所讨论的碱性强弱、溶解度、电离度、 阳离子和阴离子的影响以及有机碱和无机碱的差异之 外, 还有实验条件的影响. 目前所能买到的试剂中, 基 本都有极少量的过渡金属残余. 在多数反应中, 碱的用 量都是大过量的，这也意味着反应中的过渡金属含量也 有可能达到催化剂用量的级别, 因此对于一些反应来说 反应中所使用的碱中残留的过渡金属也可能是促进反 应发生的原因 ${ }^{[45 b, 51]}$. 对于反应中碱的选择，除了要考虑 不同碱本身的性质差异之外, 还要考虑到底物中官能团 的容忍性、反应中其他试剂对所使用碱的稳定性以及所 用的碱是否会导致其他副反应的发生等因素.

目前关于碱在过渡金属催化反应中的具体作用的研 究虽然不多, 但也已经有了一些较为详细的研究工作. 这些工作主要集中在 $\mathrm{C}-\mathrm{H}$ 键活化/官能团化反应以及氢 转移过程的研究中. 对于碱中的阳离子, 特别是碱金属、 碱土金属离子在反应中的作用, 则很少有相关研究. 而 事实上，这些金属离子可能与反应中间体有相互作用， 或是其本身就是反应活性物种的一部分, 因此它们对反

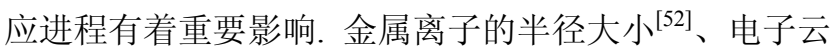
密度、与活性催化物种的相互作用方式都可能是影响反 应的原因. 由于捕捉该类中间体十分困难，目前关于这 方面的报道很少. 而未来, 对于此类中间体的研究除了 尝试实验捕捉之外，也可以多借用理论计算的方法.

\section{作者简介}

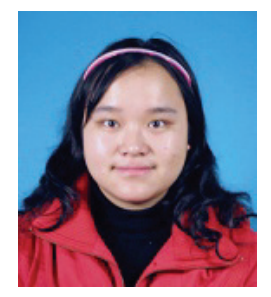

欧阳昆冰, 中国科学院化学研究所 2010 级硕博生, 参与 课题 “过渡金属催化的 $\mathrm{Si}-\mathrm{C}\left(\mathrm{sp}^{3}\right)$ 键和 $\mathrm{Silyl} \mathrm{C}\left(\mathrm{sp}^{3}\right)-\mathrm{H}$ 键的切 断反应及机理研究”. 


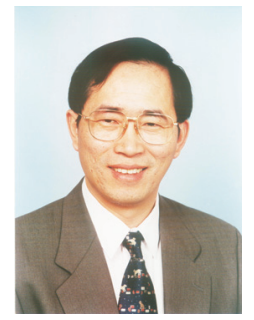

席振峰, 北京大学化学学院教授、中国科学院化学研究所 兼职研究员, 主要研究方向包括: (1)双金属有机合成试剂研究; (2)金属有机杂环试剂研究; (3)金属促进或者催化的惰性化学 键选择性切断与应用研究.

\section{References}

[1] (a) Liang, Y.; Zhang, S.; Xi, Z. J. Am. Chem. Soc. 2011, 133, 9204; (b) Liang, Y.; Geng, W.; Wei, J.; Xi, Z. Angew. Chem., Int. Ed. 2012, 51, 1934; (c) Liang, Y.; Geng, W.; Wei, J.; Ouyang, K.; Xi, Z. Org. Biomol. Chem. 2012, 10, 1537.

[2] Cella, J. A.; Bacon, S. W. J. Org. Chem. 1984, 49, 1122.

[3] http://www.acros.be/_Rainbow/pdf/brochure_cesium_v2.pdf.

[4] (a) Olmstead, W. N.; Bordwell, F. G. J. Org. Chem. 1980, 45, 3299; (b) Kuwano, R.; Utsunomiya, M.; Hartwig, J. F. J. Org. Chem. 2002, 67, 6479.

[5] Yoo, W.-J.; Capdevila, M. G.; Du, X.; Kobayashi, S. Org. Lett. 2012, 14, 5326.

[6] Yang, C.-T.; Fu, Y.; Huang, Y.-B.; Yi, J.; Guo, Q.-X.; Liu, L. Angew. Chem., Int. Ed. 2009, 48, 7398.

[7] (a) Ozawa, F.; Kubo, A.; Hayashi, T. Chem. Lett. 1992, 2177; (b) Amatore, C.; Carre, E.; Jutand, A.; M'Barki, M. Organometallics 1995, 14, 1818; (c) Amatore, C.; Carre, E.; Jutand, A.; M'Barki, M.; Meyer, G. Organometallics 1995, 14, 5605; (d) Amatore, C.; Jutand, A. Acc. Chem. Res. 2000, 33, 314.

[8] Jia, X.; Petrone, D. A.; Lautens, M. Angew. Chem., Int. Ed. 2012, 51,9870 .

[9] Zapf, A.; Beller, M. Chem. Eur. J. 2001, 7, 2908.

[10] (a) Roger, J.; Požgan, F.; Doucet, H. Adv. Synth. Catal. 2010, 352, 696; (b) Derridj, F.; Roger, J.; Djebbar, S.; Doucet, H. Org. Lett. 2010, 12, 4320.

[11] Muzart, J. Tetrahedron 2005, 61, 5955.

[12] (a) Si Larbi, K.; Fu, H. Y.; Laidaoui, N.; Beydoun, K.; Miloudi, A.; El Abed, D.; Djabbar, S.; Doucet, H. ChemCatChem 2012, 4, 815; (b) Laidaoui, N.; Roger, J.; Miloudi, A.; El Abed, D.; Doucet, H. Eur. J. Org. Chem. 2011, 4373.

[13] Lafrance, M.; Rowley, C. N.; Woo, T. K.; Fagnou, K. J. Am. Chem. Soc. 2006, 128, 8754 .

[14] Li, Y.; Wang, W.-H.; He, K.-H.; Shi, Z.-J. Organometallics 2012, $31,4397$.

[15] Duff, J. M.; Shaw, B. L. J. Chem. Soc., Dalton Trans. 1972, 2219.

[16] Duff, J. M.; Mann, B. E.; Shaw, B. L.; Turtle, B. J. Chem. Soc., Dalton Trans. 1974, 139.

[17] Gaunt, J. C.; Shaw, B. L. J. Organomet. Chem. 1975, 102, 511.

[18] Sokolov, V. I.; Troitskaya, L. L.; Reutov, O. A. J. Organomet. Chem. 1979, 182, 537.

[19] Garcia-Cuadrado, D.; Braga, A. A. C.; Maseras, F.; Echavarren, A. M. J. Am. Chem. Soc. 2006, 128, 1066.

[20] Leclerc, J.-P.; Fagnou, K. Angew. Chem., Int. Ed. 2006, 45, 7781.

[21] Garcia-Cuadrado, D.; de Mendoza, P.; Braga, A. A. C.; Maseras, F.; Echavarren, A. M. J. Am. Chem. Soc. 2007, 129, 6880.

[22] (a) González, J. J.; Garcia, N.; Gómez-Lor, B.; Echavarren, A. M. J. Org. Chem. 1997, 62, 1286; (b) Gómez-Lor, B.; Echavarren, A. M. Org. Lett. 2004, 6, 2993; (c) Pascual, S.; de Mendoza, P.; Echavarren, A. M. Org. Biomol. Chem. 2007, 2727; (d) Pascual, S.; de Mendoza, P.; Braga, A. A. C.; Maseras, F.; Echavarren, A. M. Tetrahedron 2008, 64, 6021; For a related study, see: (e) Mota, A. J.; Dedieu, A.; Bour, C.; Suffert, J. J. Am. Chem. Soc. 2005, 127, 7171.

[23] Chaumontet, M.; Piccardi, R.; Audic, N.; Hitce, J.; Peglion, J.-L.; Clot, E.; Baudoin, O. J. Am. Chem. Soc. 2008, 130, 15157.

[24] (a) Campeau, L.-C.; Bertrand-Laperle, M.; Leclerc, J.-P.; Villemure, E.; Gorelsky, S. I.; Fagnou, K. J. Am. Chem. Soc. 2008, 130, 3276; (b) Caron, L.; Campeau, L.-C.; Fagnou, K. Org. Lett. 2008, 10,
4533; (c) Gorelsky, S. I.; Lapointe, D.; Fagnou, K. J. Am. Chem. Soc. 2008, 130, 10848; (d) Lafrance, M.; Lapointe, D.; Fagnou, K. Tetrahedron 2008, 64, 6015; (e) Liégault, B.; Lapointe, D.; Caron, L.; Vlassova, A.; Fagnou, K. J. Org. Chem. 2009, 74, 1826; (f) Liégault, B.; Petrov, I.; Gorelsky, S. I.; Fagnou, K. J. Org. Chem. 2010, 75, 1047; (g) Rousseaux, S.; Gorelsky, S. I.; Chung, B. K. W.; Fagnou, K. J. Am. Chem. Soc. 2010, 132, 10692; (h) Lapointe, D.; Markiewicz, T.; Whipp, C. J.; Toderian, A.; Fagnou, K. J. Org. Chem. 2011, 76, 749; (i) Gorelsky, S. I.; Lapointe, D.; Fagnou, K. J. Org. Chem. 2012, 77, 658.

[25] (a) Ackermann, L.; Vicente, R.; Born, R. Adv. Synth. Catal. 2008, 350, 741; (b) Ackermann, L.; Vicente, R.; Althammer, A. Org. Lett. 2008, 10, 2299; (c) Zhao, D.; Wang, W.; Lian, S.; Yang, F.; Lan, J.; You, J. Chem.-Eur. J. 2009, 15, 1337; (d) Ackermann, L.; Althammer, A.; Fenner, S. Angew. Chem., Int. Ed. 2009, 48, 201; (e) Zhao, J.; Zhang, Q.; Liu, L.; He, Y.; Li, J.; Li, J.; Zhu, Q. Org. Lett. 2012, 14, 5362; (f) Guchhait, S. K.; Kandekar, S.; Kashyap, M.; Taxak, N.; Bharatam, P. V. J. Org. Chem. 2012, 77, 8321.

[26] (a) Emsley, J. J. Chem. Soc. A, 1971, 2511; (b) Clark, J. H. MS Thesis, King's College, London, 1975.

[27] Clark, J. H. Chem. Rev. 1980, 80, 429.

[28] Emsley, J.; Jones, D. J.; Miller, J. M.; Overill, R. E.; Waddilove, R. A.; J. Am. Chem. Soc. 1981, 103, 24.

[29] Clark, J. H.; Miller, J. M. J. Am. Chem. Soc. 1977, 99, 498.

[30] (a) Liotta, C. L.; Harris, H. P. J. Am. Chem. Soc. 1974, 98, 2250; (b) Naso, F.; Ronzini, L. J. Chem. Soc., Perkin Trans. 1 1974, 340; (c) Wollenberg, R. H.; Miller, S. J. Tetrahedron Lett. 1978, 19, 3219 .

[31] Denmark, S. E.; Werner, N. S. J. Am. Chem. Soc. 2010, 132, 3612.

[32] Hatanaka, Y.; Goda, K.-I.; Hiyama, T. Tetrahedron Lett. 1994, 35, 1279 .

[33] (a) Braga, A. A. C.; Morgon, N. H.; Ujaque, G.; Maseras, F. J. Am. Chem. Soc. 2005, 127, 9298; (b) Yu, D.-G.; Shi, Z.-J. Angew. Chem., Int. Ed. 2011, 50, 7097.

[34] Chen, L.; Gao, Y. Q.; Russell, D. H. J. Phys. Chem. A 2012, 116, 689.

[35] (a) Allen, L. J.; Crabtree, R. H. Green Chem. 2010, 12, 1362; (b) Liu, W.; Cao, H.; Zhang, H.; Zhang, H.; Chung, K. H.; He, C.; Wang, H.; Kwong, F. Y.; Lei, A. J. Am. Chem. Soc. 2010, 132, 16737; (c) Zhang, H.; Shi, R.; Ding, A.; Lu, L.; Chen, B.; Lei, A. Angew. Chem., Int. Ed. 2012, 51, 12542; (d) Zhang, J.; Bellomo, A.; Creamer, A. D.; Dreher, S. D.; Walsh, P. J. J. Am. Chem. Soc. 2012, 134,13765

[36] Chen, I.-H.; Yin, Y.; Itano, W.; Kanai, K.; Shibasaki, M. J. Am. Chem. Soc. 2009, 131, 11664.

[37] Yazaki, R.; Nitabaru, T.; Kumagai, N.; Shibasaki, M. J. Am. Chem. Soc. 2008, 130, 14477.

[38] Campeau, L.-C.; Parisien, M.; Jean, A.; Fagnou, K. J. Am. Chem. Soc. 2006, 128, 581.

[39] Chen, L.; Roger, J.; Bruneau, C.; Dixneuf, P. H.; Doucet, H. Adv. Synth. Catal. 2011, 353, 2749.

[40] Shirakawa, E.; Itoh, K.-I.; Higashino, T.; Hayashi, T. J. Am. Chem. Soc. 2010, 132, 15537.

[41] For reviews on cation- $\pi$ interaction, see: (a) Ma, J. C.; Dougherty, D. A. Chem. Rev. 1997, 97, 1303; (b) Gokel, G. W.; De Wall, S. L.; Meadows, E. S. Eur. J. Org. Chem. 2000, 2967.

[42] (a) Robinson, J. R.; Carroll, P. J.; Walsh, P. J.; Schelter, E. J. Angew. Chem., Int. Ed. 2012, 51, 10159; (b) Beweries, T.; Burlakov, V. V.; Bach, M. A.; Peitz, S.; Arndt, P.; Baumann, W.; Spannenberg, A.; Rosenthal, U.; Pathak, B.; Jemmis, E. D. Angew. Chem., Int. Ed. 2007, 46, 6907 .

[43] Chowdhury, R. L.; Bäckvall, J.-E. J. Chem. Soc., Chem. Commun. 1991, 1063.

[44] Morto, D.; Cole-Hamilton, D. J. J. Chem. Soc., Chem. Commun. 1988, 1154

[45] (a) Polshettiwar, V.; Varma, R. S. Green Chem. 2009, 11, 1313; (b) Ouali, A.; Majoral, J.-P.; Caminade, A.-M.; Taillefer, M. ChemCatChem 2009, 1, 504.

[46] (a) Qafisheh, N.; Mukhopadhyay, S.; Joshi, A. V.; Sasson, Y.; Chuah, G.-K.; Jaenicke, S. Ind. Eng. Chem. Res. 2007, 46, 3016; (b) Popov, I.; Do, H. Q.; Daugulis, O. J. Org. Chem. 2009, 74, 8309; (c) Gerber, R.; Blacque, O.; Frech, C. M. ChemCatChem. 2009, 1, 393; (d) Lamblin, M.; Nassar-Hardy, L.; Hierso, J.-C.; Fouquet, E.; Felpin, F.-X. Adv. Synth. Catal. 2010, 352, 33; (e) Radhakrishan, R.; Do, D. M.; Jaenicke, S.; Sasson, Y.; Chuah, G.-K. ACS Catal. 2011, 
1,1631 .

[47] (a) Pearson, R. G. J. Am. Chem. Soc. 1963, 85, 3533; (b) Chen, J.-P.; Peng, Q.; Lei, B.-L.; Hou, X.-L.; Wu, Y.-D. J. Am. Chem. Soc. 2011, 133, 14180; (c) Liu, J.; Hu, J. Chem. Eur. J. 2010, 16, 11443; (d) Shen, X.; Zhang, L.; Zhao, Y.; Zhu, L.; Li, G.; Hu, J. Angew. Chem., Int. Ed. 2011, 50, 2588.

[48] (a) De Graauw, C. F.; Peters, J. A.; van Bekkum, H.; Huskens, J. Synthesis 1994, 10, 1007; (b) Cha, J. S. Org. Process Res. Dev. 2006, 10, 1032.
[49] Zheng, D.; Li, S.; Wu, J. Org. Lett. 2012, 14, 2655.

[50] (a) Durola, F.; Durot, S.; Heitz, V.; Joosten, A.; Sauvage, J.-P.; Trolez, Y. J. Incl. Phenom. Macrocycl. Chem. 2011, 71, 507. For a similar result, also see: (b) Cariou, M.; Simonet, J. J. Chem. Soc., Chem. Commun. 1984, 1146.

[51] Sun, C.-L.; Li, H.; Yu, D.-G.; Yu, M.; Zhou, X.; Lu, X.-Y.; Huang, K.; Zheng, S.-F.; Li, B.-J.; Shi, Z.-J. Nat. Chem. 2010, 2, 1044.

[52] Xie, J.-H.; Liu, S.; Huo, X.-H.; Cheng, X.; Duan, H.-F.; Fan, B.-M.; Wang, L.-X.; Zhou, Q.-L. J. Org. Chem. 2005, 70, 2967.

(Zhao, C.) 